Pacific Journal of Mathematic 


\section{SUBDIRECT SUMS AND INFINITE ABELIAN GROUPS}

\section{ELBERT A. WALKER}

1. Definitions. Let $G$ be a group, and suppose $G$ is a subgroup of the direct sum $\sum_{a \in I} \oplus H_{a}$ of the collection of groups $\left\{H_{a}\right\}_{a \in I}$. If the projection of $G$ into $H_{a}$ is onto $H_{a}$ for each $a \in I$, then $G$ is said to be a subdirect sum of the groups $\left\{H_{a}\right\}_{a \in I}$. (Only weak direct and subdirect sums are considered here.) If a group $G$ is isomorphic to a subdirect sum of the groups $\left\{H_{a}\right\}_{a \in I}$, then $G$ is said to be represented as a subdirect sum of the groups $\left\{H_{a}\right\}_{a \in I}$. A group is called a rational group if it is a subgroup of a $Z\left(p^{\infty}\right)$ group or a subgroup of the additive group of rational numbers.

2. THEOREM. Every Abelian group can be represented as a subdirect sum of rational groups where the subdirect sum intersects each of the rational groups non-trivially.

Proof. $G$ is isomorphic to a subgroup of some divisible group, and thus can be represented as a subdirect sum $G^{\prime}$ of rational group $\left\{H_{a}\right\}_{a \in I}$. Let $\left(h_{1}, h_{2}, \cdots, h_{a}, \cdots\right)$ be an element of $G^{\prime}$. Let $\left(h_{1}, h_{2}, \cdots, h_{a}, \cdots\right) \beta_{1}=$ $\left(k_{1}, h_{2}, \cdots, h_{a}, \cdots\right)$, where $k_{1}=h_{1}$ if $G^{\prime} \cap H_{1} \neq 0$, and $k_{1}=0$ if $G^{\prime} \cap H_{1}=0$. Assume $\beta_{c}$ has been defined for $c<b$. Define

$$
\left(h_{1}, h_{2}, \cdots, h_{a}, \cdots\right) \beta_{b}=\left(k_{1}, k_{2}, \cdots, k_{b}, h_{b+1}, \cdots\right)
$$

where $k_{b}=h_{b}$ if $H_{b} \cap\left(\bigcup_{c<b} G^{\prime} \beta_{c}\right) \neq 0$, and $k_{b}=0$ otherwise. Each $\beta_{a}$ preserves addition because each is a projection. Let $\left(h_{1}, h_{2}, \cdots, h_{a}, \cdots\right) \neq$ $(0,0, \cdots, 0, \cdots)$ and let

$$
\left(h_{1}, h_{2}, \cdots, h_{a}, \cdots\right) \beta_{a}=\left(k_{1}, k_{2}, \cdots, k_{a}, h_{a+1}, h_{a+2}, \cdots\right) .
$$

Only a finite number of the coordinates of $\left(h_{1}, h_{2}, \cdots, h_{a}, \cdots\right)$ are not 0 . Let them be $h_{a_{1}}, h_{a_{2}}, \cdots, h_{a_{n}}$, where $a_{1}<a_{2}<\cdots<a_{n}$. If $a<a_{n}$, then

$$
\begin{aligned}
& \left(h_{1}, h_{2}, \cdots, h_{a}, \cdots\right) \beta_{a} \\
& \quad=\left(k_{1}, k_{2}, \cdots, k_{a}, h_{a+1}, \cdots, h_{a_{n}}, h_{a_{n}+1}, \cdots\right) \neq(0,0, \cdots, 0, \cdots)
\end{aligned}
$$

since $h_{a_{n}} \neq 0$. Assume $a \geqq a_{n}$. If $n=1$ and $a_{1}=1$, then $\left(h_{1}, h_{2}, \cdots, h_{a}, \cdots\right)=$ $\left(h_{a_{1}}, 0,0, \cdots, 0, \cdots\right) \in G^{\prime}$ and $G^{\prime} \cap H_{1} \neq 0$ so that $\left(h_{a_{1}}, 0,0, \cdots,{ }^{n}\right.$ is $=$ $\left(h_{a_{1}}, 0,0, \cdots, 0, \cdots\right)$. That is, $k_{a_{1}}=h_{a_{1}} \neq 0$, and hence $\left(h_{1}, h_{2}, \cdots: \quad \neq\right.$ $(0,0, \cdots, 0, \cdots)$. If $n=1$ and $a_{n} \neq 1$, then $\left(0,0, \cdots, h_{a_{1}}, 0,0, \cdots\right) \in G^{\prime}$ and also in $G^{\prime} \beta_{c}$ for all $c<a_{1}$. Thus $H_{a_{1}} \cap\left(\mathbf{U}_{c<a_{1}} G^{\prime} \beta_{c}\right) \neq 0$, and

Received September 19, 1958, in revised form October 20, 1958. 


$$
\begin{aligned}
& \left(h_{1}, h_{2}, \cdots, h_{a}, \cdots\right) \beta_{a}=\left(0,0, \cdots, 0, h_{a_{1}}, 0,0, \cdots\right) \beta_{a} \\
& \quad=\left(0,0, \cdots, 0, h_{a_{1}}, 0,0, \cdots\right) \beta_{a_{1}}=\left(0,0, \cdots 0, h_{a_{1}}, 0,0, \cdots\right) \\
& \quad \neq(0,0, \cdots, 0, \cdots) .
\end{aligned}
$$

Assume $n>1$. If $\left(h_{1}, h_{2}, \cdots, h_{a}, \cdots\right) \beta_{a}=(0,0, \cdots, 0, \cdots)$, then $k_{c}=0$ for $c \leqq a_{n}$, and

$$
\left(h_{1}, h_{2}, \cdots, h_{a}, \cdots\right) \beta_{a_{n-1}}=\left(0,0, \cdots, 0, h_{a_{n}}, 0,0, \cdots\right) .
$$

Therefore $H_{a_{n}} \cap\left(G^{\prime} \beta_{a_{n-1}}\right) \neq 0$, and so $H_{a_{n}} \cap\left(\bigcup_{c<a} G^{\prime} \beta_{c}\right) \neq 0$. Hence $k_{a_{n}}=$ $h_{a_{n}} \neq 0$, and this contradicts $k_{c}=0$ for $c \leqq a_{n}$. Therefore

$$
\left(h_{1}, h_{2}, \cdots, h_{a}, \cdots\right) \beta_{a} \neq(0,0, \cdots, 0, \cdots),
$$

and the kernel of $\beta_{a}$ is 0 . Hence each $\beta_{a}$ is an isomorphism. Now let $\left(h_{1}, h_{2}, \cdots, h_{a}, \cdots\right) \beta=\left(k_{1}, k_{2}, \cdots, k_{a}, \cdots\right)$. Clearly $\beta$ is a homomorphism of $G^{\prime}$ into $\sum_{a \in I} \oplus H_{a}$. But the kernel of $\beta$ is 0 because every element in $G^{\prime}$ has only a finite number of non-zero coordinates. Let $I^{\prime}$ be the set of indices such that $a \notin I^{\prime}$ implies that the image of the projection of $G^{\prime} \beta$ into $H_{a}$ is $0 . \quad G^{\prime} \beta$ is isomorphic to a subdirect sum of the groups $\left\{H_{a}\right\}_{a \in I^{\prime}}$. If $G^{\prime} \beta \cap H_{1}=0$, then for $\left(h_{1}, h_{2}, \cdots, h_{a}, \cdots\right) \in G^{\prime}$ we have $\left(h_{1}, h_{2}, \cdots, h_{a}, \cdots\right) \beta_{1}=\left(0, h_{2}, \cdots, h_{a}, \cdots\right)$, so that

$$
\left(h_{1}, h_{2}, \cdots, h_{a}, \cdots\right) \beta=\left(0, k_{2}, k_{3}, \cdots, k_{a}, \cdots\right) .
$$

Hence the image of the projection of $G^{\prime} \beta$ into $H_{1}$ is 0 . Therefore $1 \notin I^{\prime}$. Let $a>1$. Suppose $G^{\prime} \beta \cap H_{a}=0$ and $H_{a} \cap\left(\bigcup_{c<a} G^{\prime} \beta_{c}\right) \neq 0$. Then there exists $b<a$ such that $H_{a} \cap G^{\prime} \beta_{b} \neq 0$. Let $\left(0,0, \cdots, 0, k_{a}, 0,0, \cdots\right) \in H_{a} \cap G^{\prime} \beta_{b}$, where $k_{a} \neq 0$. Let $\left(h_{1}, h_{2}, \cdots, h_{a}, \cdots\right) \beta_{b}=\left(0,0, \cdots, 0, k_{a}, 0,0, \cdots\right)$. Then $\left(h_{1}, h_{2}, \cdots, h_{a}, \cdots\right) \beta=\left(0,0, \cdots, 0, k_{a}, 0,0, \cdots\right)$, and so $G^{\prime} \beta \cap H_{a} \neq 0$. Therefore if $G^{\prime} \beta \cap H_{a}=0$, then $H_{a} \cap\left(\bigcup_{c<a} G^{\prime} \beta_{c}\right)=0$. This implies for every $\left(h_{1}, h_{2}, \cdots h_{a}, \cdots\right) \in G^{\prime}$ that

$$
\left(h_{1}, h_{2}, \cdots, h_{a}, \cdots\right) \beta_{a}=\left(k_{1}, k_{2}, \cdots, k_{a}, h_{a+1}, h_{a+2}, \cdots\right),
$$

where $k_{a}=0$, and hence that

$$
\left(h_{1}, h_{2}, \cdots, h_{a}, \cdots\right) \beta=\left(k_{1}, k_{2}, \cdots, 0, k_{a+1}, k_{a+2}, \cdots\right) .
$$

Thus the image of the projection of $G^{\prime} \beta$ into $H_{a}$ is 0 so that $a \notin I^{\prime}$. Hence for $a \in I^{\prime}, G^{\prime} \beta \cap H_{a} \neq 0$. Since $G$ is isomorphic to $G^{\prime} \beta$, the theorem follows.

3. REMARKS. Theorem 9 in [1] is an immediate corollary of the preceding theorem, as are some other known theorems in Abelian group theory. In [2], Scott proves that every uncountable Abelian group $G$ has, for every possible infinite index $\alpha, 2^{o(G)}$ subgroups of order equal to $o(G)$ and of index $\alpha$, and that for each given infinite index, their intersection is 0 . The following theorem shows that if $G$ is torsion free, one can say more. 
4. THEOREM. Every torsion free Abelian group $G$ of infinite rank has, for every possible infinite index $\alpha, 2^{o(G)}$ pure subgroups of order equal to $o(G)$ and of index $\alpha$. Furthermore, the intersection of these pure subgroups of index $\alpha$ is 0 .

Proof. Represent $G$ as a subdirect sum $G^{\prime}$ of rational groups $\left\{H_{a}\right\}_{a \in I}$ such that for each $a \in I, G^{\prime} \cap H_{a} \neq 0$. Let $\alpha$ be an infinite cardinal such that $\alpha \leqq o(G) . o(I)=o(G)$ since $G$ has infinite rank. Let $I=S_{1} \cup S_{2}$ where $o\left(S_{1}\right)=\alpha, o\left(S_{2}\right)=o(G)$, and $S_{1} \cap S_{2}=\phi$. Let $T$ be a subset of $S_{2}$ such that $o\left(S_{2}-T\right)=o(G)$. There are $2^{o(G)}$ such $T^{\prime \prime} s$. Let $\left(h_{1}, h_{2}, \cdots, h_{a}, \cdots\right)$ be in $G^{\prime}$, and let

$$
\left(h_{1}, h_{2}, \cdots, h_{a}, \cdots\right) t=\left(\sum_{j \in T} h_{j}, k_{1}, k_{2}, \cdots, k_{a}, \cdots\right),
$$

where $k_{i}=h_{i}$ if $i \in S_{1}$ and $k_{i}=0$ otherwise. The mapping $t$ is a homomorphism and the order of its image is equal to $o\left(S_{1}\right)$. That is, the index of the kernel of $t$ is $\alpha$. The order of the kernel of $t$ is equal to $o(G)$ since $o\left(S_{2}-T\right)=o(G)$, and $G^{\prime} \cap H_{a} \neq 0$ for all $a \in I$. Let $T, T^{\prime} \supseteqq S_{2}$, $T \neq T^{\prime}$. Then there is a $j \in T$ such that $j \notin T^{\prime}$, say. Let $h_{j} \in G^{\prime}, h_{j} \neq 0$. Then

$$
\left(0,0, \cdots, h_{\jmath}, 0,0, \cdots\right) t=\left(h_{\jmath}, 0, \cdots\right) .
$$

However, $\left(0,0, \cdots, h_{y}, 0,0, \cdots\right) t^{\prime}=(0,0,0, \cdots)$. Hence the kernel of $t$ is not the same as the kernel of $t^{\prime}$. These kernels are pure in $G^{\prime}$ since the quotient groups are torsion free. Thus $G$ has $2^{o(\theta)}$ pure subgroups of index $\alpha$, and of order equal to $o(G)$. Suppose $\left(h_{1}, h_{2}, \cdots, h_{a}, \cdots\right)$ is in the intersection of all these pure subgroups of index $\alpha$. Then if $b \in S_{1}, h_{b}=0$. Hence if $h_{c} \neq 0$, letting $T=\{c\}$, we have

$$
\left(h_{1}, h_{2}, \cdots, h_{c}, \cdots, h_{a}, \cdots\right) t=\left(h_{c}, 0,0, \cdots\right) \neq 0,
$$

which is impossible. Therefore for each $a \in I, h_{a}=0$, and this shows that the intersection of these subgroups is 0 .

5. REMarks. Every torsion free divisible group $D$ of rank $\alpha$ is a direct sum of $\alpha$ copies of the additive group of rational numbers, and $D$ contains an isomorphic copy of every torsion free Abelian group of rank $\alpha$. The following theorem says that if $\alpha$ is infinite, every torsion free Abelian group of rank $\alpha$ is represented in a special way in $D$.

6. THEOREM. Every torsion free Abelian group $G$ of infinite rank can be represented as a subdirect sum $G^{\prime}$ of copies of the additive group of rational numbers, and in such a way that $G^{\prime}$ intersects each subdirect summand non-trivially.

Proof. Represent $G$ as a subdirect sum $G^{\prime}$ of the rational groups 
$\left\{H_{a}\right\}_{a \in I}$ such that for each $a \in I, G^{\prime} \cap H_{a} \neq 0$. Suppose first that $G$ has countably infinite rank. That is, suppose $I$ is the set of positive integers. Each $H_{a}$ is a subgroup of the additive group of rational numbers, since $G$ is torsion free. Let $k_{1}, k_{2}, k_{3}, \cdots$ be a sequence of non-zero rational numbers such that $k_{i} \in G^{\prime} \cap H_{i}$. Let $r_{1}, r_{2}, r_{3}, \cdots$ be the nonzero rational numbers arranged in a sequence. Let $s_{i}=r_{i} / k_{i}$. Let $\left(h_{1}, h_{2}, \cdots, h_{n}, \cdots\right)$ be an element of $G^{\prime}$. Let

$$
\left(h_{1}, h_{2}, \cdots, h_{n}, \cdots\right) \beta=\left(\sum_{i=1}^{\infty} s_{i} h_{i}, \sum_{i=2}^{\infty} s_{i} h_{i}, \cdots, \sum_{i=n}^{\infty} s_{i} h_{i}, \cdots\right) .
$$

Since only a finite number of the $h_{i}$ 's are non-zero, for each $k, \sum_{i=k}^{\infty} s_{i} h_{i}$ is a rational number, and for only a finite number of $k$ 's is $\sum_{i=k_{i}}^{\infty} s_{i} h_{i}$ nonzero.

$$
\begin{aligned}
& \left(\left(h_{1}, h_{2}, \cdots, h_{n}, \cdots\right)+\left(g_{1}, g_{2}, \cdots, g_{n}, \cdots\right)\right) \beta \\
& \quad=\left(h_{1}+g_{1}, h_{2}+g_{2}, \cdots, h_{n}+g_{n}, \cdots\right) \beta \\
& \quad=\left(\sum_{i=1}^{\infty} s_{i}\left(h_{i}+g_{i}\right), \cdots, \sum_{i=n}^{\infty} s_{i}\left(h_{i}+g_{i}\right), \cdots\right) \\
& \quad=\left(\sum_{i=1}^{\infty} s_{i} h_{i}+\sum_{i=1}^{\infty} s_{i} g_{i}, \cdots, \sum_{i=n}^{\infty} s_{i} h_{i}+\sum_{i=n}^{\infty} s_{i} g_{i}, \cdots\right) \\
& \quad=\left(h_{1}, h_{2}, \cdots, h_{n}, \cdots\right) \beta+\left(g_{1}, g_{2}, \cdots, g_{n}, \cdots\right) \beta .
\end{aligned}
$$

Hence $\beta$ is a homomorphism of $G^{\prime}$ into a direct sum of copies of the additive group $R$ of rationals. Let $R_{n}$ be the set of $n$th coordinates of elements of $G^{\prime} \beta . \quad R_{n}$ is a subgroup of $R$ since it is the image of the projection of $G^{\prime} \beta$ onto its $n$th coordinates. Let $m \geqq n$.

$$
\left(0,0, \cdots, 0, k_{m}, 0,0, \cdots\right) \in G^{\prime}
$$

and

$$
\left(0,0, \cdots, 0, k_{m}, 0,0, \cdots\right) \beta=\left(r_{m}, r_{m}, \cdots, r_{m}, 0,0, \cdots\right),
$$

so that $r_{m} \in R_{n}$. Thus $R_{n}$ contains all but at most a finite number of elements of $R$, and being a subgroup of $R$, must then be $R$. Therefore $G^{\prime} \beta$ is a subdirect sum of copies of $R$. Let $x \in G^{\prime}, x \neq 0$, and let $h_{r}$ be the last non-zero coordinate of $x$. Then the $r$ th coordinate of $x \beta$ is $s_{r} h_{r} \neq 0$. Hence the kernel of $\beta$ is 0 and $\beta$ is an isomorphism of $G$ onto a subdirect sum of copies of $R$. Now consider the case where $I$ is not countable. Let $I$ be the union of the set of mutually disjoint countably infinite sets $\left\{I_{j}\right\}_{j \in J}$. Denote by $S_{j}$ the image of the projection of $G^{\prime}$ into $\sum_{a \in I_{j}} \oplus H_{a}$. Then $G^{\prime}$ is a subdirect sum of the set of groups $\left\{S_{j}\right\}_{j \in J}$, and each $S_{j}$ is of countably infinite rank. Hence each $S_{j}$ may be represented as a subdirect sum of copies of the additive group of rational numbers, and it follows that $G$ may be so represented. In light of the proof of 2 , this representation may be assumed to intersect each subdirect summand non-trivially. 


\section{REFERENCES}

1. W. R. Scott, Groups, and cardinal numbers, Amer. J. Math. 74 (1952), 187-197.

2. W. R. Scott, The number of subgroups of given index in nondenumerable Abelian groups, Proc. Amer. Math. Soc. 5 (1954), 19-22.

UNIVERSITY OF KANSAS AND

NeW Mexico State University 



\section{PACIFIC JOURNAL OF MATHEMATICS}

\section{EDITORS}

\section{David Gilbarg}

Stanford University

Stanford, California

R. A. Beaumont

University of Washington

Seattle 5 , Washington
A. L. Whiteman

University of Southern California

Los Angeles 7, California

L. J. PAIGE

University of California

Los Angeles 24, California

\section{ASSOCIATE EDITORS}
E. F. BECKENBACH
C. E. BURGESS
E. HEWITT
A. HORN

\author{
V. GANAPATHY IYER \\ R. D. JAMES \\ M. S. KNEBELMAN \\ L. NACHBIN
}
I. NIVEN
E. G. STRAUS
T. G. OSTROM
H. L. ROYDEN
G. SZEKERES
M. M. SCHIFFER
F. WOLF
K. YOSIDA

\section{SUPPORTING INSTITUTIONS}

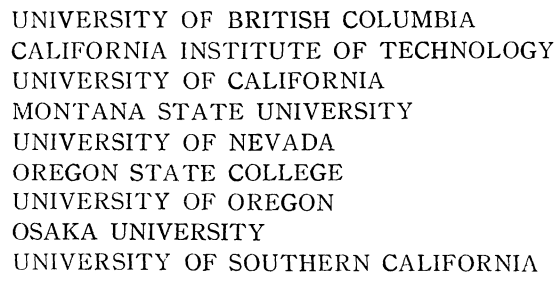

UNIVERSITY OF BRITISH COLUMBIA CALIFORNIA INSTITUTE OF TECHNOLOGY UNIVERSITY OF CALIFORNIA MONTANA STATE UNIVERSITY UNIVERSITY OF NEVADA OREGON STATE COLLEGE UNIVERSITY OF OREGON OSAKA UNIVERSITY UNIVERSITY OF SOUTHERN CALIFORNIA

STANFORD UNIVERSITY

UNIVERSITY OF TOKYO

UNIVERSITY OF UTAH

WASHINGTON STATE COLLEGE

UNIVERSITY OF WASHINGTON

AMERICAN MATHEMATICAL SOCIETY CALIFORNIA RESEARCH CORPORATION HUGHES AIRCRAFT COMPANY SPACE TECHNOLOGY LABORATORIES

Printed in Japan by Kokusai Bunken Insatsusha (International Academic Printing Co., Ltd.), Tokyo, Japan 


\section{Pacific Journal of Mathematics}

\section{Vol. 9, No. 1 \\ May, 1959}

Julius Rubin Blum and Murray Rosenblatt, On the structure of infinitely

divisible distributions . ............................. 1

Robert Geroge Buschman, Asymptotic expressions for

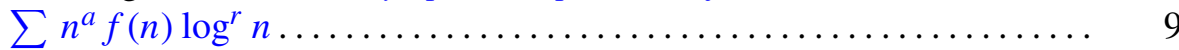

Eckford Cohen, A class of residue systems $(\bmod r)$ and related arithmetical

functions. I. A generalization of Möbius inversion .............. 13

Paul F. Conrad, Non-abelian ordered groups ................... 25

Richard Henry Crowell, On the van Kampen theorem............... 43

Irving Leonard Glicksberg, Convolution semigroups of measures ........ 51

Seymour Goldberg, Linear operators and their conjugates ............ 69

Olof Hanner, Mean play of sums of positional games .............. 81

Erhard Heinz, On one-to-one harmonic mappings ................ 101

John Rolfe Isbell, On finite-dimensional uniform spaces . . ........... 107

Erwin Kreyszig and John Todd, On the radius of univalence of the function

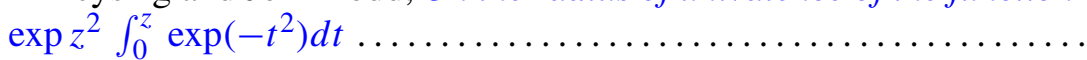

Roger Conant Lyndon, An interpolation theorem in the predicate

calculus......................................... 129

Roger Conant Lyndon, Properties preserved under homomorphism ........ 143

Roger Conant Lyndon, Properties preserved in subdirect products ....... 155

Robert Osserman, A lemma on analytic curves ................ 165

R. S. Phillips, On a theorem due to Sz.-Nagy..................... 169

Richard Scott Pierce, A generalization of atomic Boolean algebras ....... 175

J. B. Roberts, Analytic continuation of meromorphic functions in valued fields................................. 183

Walter Rudin, Idempotent measures on Abelian groups ................ 195

M. Schiffer, Fredholm eigen values of multiply-connected domains ........ 211

V. N. Singh, A note on the computation of Alder's polynomials ......... 271

Maurice Sion, On integration of 1-forms ...................... 277

Elbert A. Walker, Subdirect sums and infinite Abelian groups........... 287

John W. Woll, Homogeneous stochastic processes . .................. 293 